\title{
First Relapse of Acute Lymphoblastic Leukemia in Children in Upper Egypt: Survival Outcome and Prognostic Factors
}

\author{
Azza Shibl ${ }^{1}$, Heba A. Sayed ${ }^{1}$, Asmaa M. Zahran ${ }^{2}$ \\ ${ }^{1}$ Pediatric Oncology Department, South Egypt Cancer Institute, Assiut University, Assiut, Egypt; \\ ${ }^{2}$ Clinical Pathology Department, South Egypt Cancer Institute, Assiut University, Assiut, Egypt
}

\begin{abstract}
Background: Relapse is the main reason of treatment failure in childhood acute Lymphoblastic leukemia (ALL). Aim: To study the treatment outcome of first ALL relapse in response to two different reinduction regimens and prognostic factors predicting outcome.

Methods: A retrospective study that included 82 children with ALL in the $1^{\text {st }}$ relapse from two tertiary oncology centers in Upper Egypt. Patients were treated according to the St. Jude ALL-R16 protocol. Seventeen patients were treated with a standard reinduction (regimen 1) and 65 were treated with a modified reinduction regimen in which anthracycline was added and asparaginase was reduced to 9 doses (regimen 2). Response, survival and prognostic factors were analyzed.

Results: Second, complete remission (CR2) was achieved in 57\% of all patients (65\% with regimen 2 vs. $29 \%$ with regimen $1, p=0.009$ ). FLAG regimen resulted in achieving CR2 in all patients with reinduction failure. Treatment related mortality was more common with regimen 2 than with regimen 1 ( $34 \%$ vs. $12 \%$, respectively). For all patients, the 2-year overall and event-free survival rates were $30 \%$ and $25 \%$. In multivariate analysis, high initial total leukocytic count, isolated medullary relapse, regimen 1 and very early relapse were independently associated with worse event free survival ( $\mathrm{p}=0.031,0.017,0.037$ and 0.001 ; respectively).

Conclusions: The overall outcome of treatment of first ALL relapse in children in our region is poor. New intensive chemotherapy regimens may help in improving the treatment outcome.
\end{abstract}

Keywords: Acute Lymphoblastic Leukemia, First relapse, Children, Survival, Prognostic factors, Egypt

Corresponding author: Azza Shibl, MD; Pediatric Oncology Department, South Egypt Cancer Institute, Assiut University, Assiut, Egypt; Email: azzashibl@aun.edu.eg

Submitted: 9-May-2021, Finally revised: 15-August-2021, Accepted: 19-September-2021, Published online: 30-January-2022

\section{Introduction}

During the past decades, marked treatment improvement in acute lymphoblastic leukemia (ALL) has been achieved ${ }^{1,2}$. However, relapse is still the major cause for treatment failure and its outcome hasn't improved largely ${ }^{3-5}$.

Treatment of ALL relapse in countries with limited resources is further complicated because of the limited availability of salvage treatment the and lack of chance of bone marrow transplantation (BMT) or target therapy. In addition to other factors such as the high treatment-related mortality and lack of awareness ${ }^{6}$.

In previous salvage therapy protocols, reinduction therapy was mostly similar to the induction therapy of frontline protocols and the policies for post-remission therapy were not uniform and the outcomes were poor $3,7,8$. At St. Jude Children's Research Hospital (SJCRH), the use of intensified rotational combined chemotherapy in relapsing ALL protocol avoids the development of drug resistance through a rapid succession of the use of multiple agents. Moreover, fludarabine, a fluorinated purine analogue that isn't incorporated in the frontline protocols of pediatric ALL, has a great success in consolidating a second remission 9, 10. Also, UK-ALLR3 study reported a significant benefit of mitoxantrone in progression-free and overall survival (OS) in children with relapsed ALL based on its chemosensitivity profile especially Bcell through different mechanisms ${ }^{11}$. Other anthracyclines, such as daunorubicin and 
doxorubicin, showed their efficacy in relapsing ALL patients in different BFM treatment protocols ${ }^{5,8}$.

Previously at our institution, there was no uniform protocol for treating relapsed ALL patients. With the use of the St. Jude ALL TXIIIB protocol, survival of newly diagnosed pediatric ALL improved at our institution while the relapsed patients died shortly after relapse ${ }^{12}$. We aimed at assessing the outcome of ALL children in first relapse treated with the St. Jude ALL R16 protocol with the addition of an anthracycline during reinduction therapy. Additional aims were to confirm the established prognostic factors and to evaluate new ones in the light of this treatment strategy.

\section{Methods}

This retrospective study included 82 pediatric patients who presented at Pediatric Oncology Departments of South Egypt Cancer Institute and Sohag Cancer Center with first ALL relapse (isolated medullary relapse [IMR], isolated extramedullary relapse [IEMR], or combined) from October 2013 to May 2019. Relapse with lineage switch or subsequent relapses were excluded. In addition, patients who died within one week of admission before starting effective therapy or those who abandoned initial treatment were excluded.

Patients' records were reviewed for the diagnosis of first ALL relapse, site, and timing of relapse in addition to patients' characteristics at initial diagnosis. We classified the timing of relapse into very early if relapse occurred within 18 months of diagnosis; early if occurred $\geq 18$ months of diagnosis till 6 months after finishing treatment and late if relapse occurred after 6 months from finishing ${ }^{13}$.

Patients were treated according to the St. Jude ALL- R16 protocol. Reinduction in 17 patients was as the original protocol (regimen 1) ${ }^{9}$. An anthracycline was added on days 1 and 8 of reinduction therapy in 65 patients (regimen 2) as follow: patients with very early / early relapse received mitoxantrone (12 $\left.\mathrm{mg} / \mathrm{m}^{2}\right)$ or daunorubicin $\left(25 \mathrm{mg} / \mathrm{m}^{2}\right)$ and patients with late relapse received daunorubicin $\left(25 \mathrm{mg} / \mathrm{m}^{2}\right)$. Asparaginase in regimen 2 was given for 9 doses instead of 12 doses as in regimen 1 . After reinduction, all patients were continued according to the original protocol, including primary consolidation (FLAG [fludarabine, cytarabine, and granulocyte colony-stimulating factor]), secondary consolidation, then 2 modules of four cycles of intensive continuation and lastly 17 months of standard continuation. Teniposide in the standard continuation was substituted by etoposide $(200 \mathrm{mg} /$ $\mathrm{m}^{2}$ ) due to its unavailability.

Second complete remission (CR2) of bone marrow relapse was defined as $<5 \%$ blasts in the recovered bone marrow in the absence of clinical signs of disease. Patients who did not achieve CR2 were considered reinduction failure and surviving patients were termed refractory ${ }^{14}$, but they continued the treatment protocol. Disease-related mortality included deaths during reinduction failure or $2^{\text {nd }}$ relapse even if treatment toxicity was the direct cause. Treatment-related mortality (TRM) included deaths due to treatment toxicity and $2^{\text {nd }}$ malignancy ${ }^{15}$.

\section{Statistical analysis}

Patients were followed up until august 2020. Median and range were used to describe the continuous data and number and percentage for the categorical data. Chi-square / Fisher exact test was used to compare proportions. For survival analysis, Overall survival was calculated from the date of diagnosis of first relapse to the date of death due to any cause. Event-free survival (EFS) was calculated from the date of first relapse to the date of death of any cause, reinduction failure, second relapse, or occurrence of a $2^{\text {nd }}$ malignancy. Patients with reinduction failure were considered as having an event on day $0^{16}$. Kaplan-Meier method was used to estimate both OS and EFS, and comparison between groups was made using the log-rank test. Variables, that were significant in univariate analysis, were included in multivariate cox regression analysis to find the independent prognostic factors affecting EFS after relapse. P-value was always two-tailed and significant at 0.05 level. The IBM SPSS software, version 23.0. (Armonk, NY: IBM Corp.) was used for data management.

\section{Results}

\section{Patients' characteristics}

Eighty-two patients with ALL in the first relapse were eligible for this study. The median time to relapse was 27 months (range: 3-100). The initial frontline treatment included modified TXIII-B protocol ${ }^{17}$ (75 patients), BFM-90 (5 patients) ${ }^{18}$, or TXV (2 patient) ${ }^{19}$. Patients' characteristics in relation to the timing of relapse are summarized in Table 1. Sixteen (19.5\%) patients relapsed late and 66 (80.5\%) patients had an early relapse, 24 of them occurred very early. Older patients at diagnosis ( $\geq 10$ years) and those with T-cell ALL relapsed very early or 
Table 1: Characteristics of 82 children with acute lymphoblastic leukemia in $1^{\text {st }}$ relapse

\begin{tabular}{|c|c|c|c|c|c|}
\hline Variable & $\begin{array}{l}\text { Total } \\
(n=82)\end{array}$ & $\begin{array}{l}\text { Very early } \\
\text { relapse } \\
(n=24)\end{array}$ & $\begin{array}{l}\text { Early } \\
\text { relapse } \\
(n=42)\end{array}$ & $\begin{array}{l}\text { Late } \\
\text { relapse } \\
(n=16)\end{array}$ & $p$ value \\
\hline \multicolumn{6}{|l|}{ Age at diagnosis (years) } \\
\hline Median (range) & 7 (1-16.5) & $10.5(3-16.5)$ & $9(1-16)$ & $5(1.5-9)$ & \\
\hline$<10$ & $51(62.2 \%)$ & $10(41.7 \%)$ & $25(59.5 \%)$ & $16(100 \%)$ & \multirow[t]{2}{*}{0.001} \\
\hline$\geq 10$ & $31(37.8 \%)$ & $14(58.3 \%)$ & $17(40.5 \%)$ & 0 & \\
\hline \multicolumn{6}{|l|}{ Sex } \\
\hline Male & $40(48.8 \%)$ & $15(62.5 \%)$ & $19(45.2 \%)$ & $6(37.6 \%)$ & \multirow[t]{2}{*}{0.242} \\
\hline Female & $42(51.2 \%)$ & $9(37.5 \%)$ & $23(54.8 \%)$ & $10(62.5 \%)$ & \\
\hline \multicolumn{6}{|l|}{ TLC at diagnosis $\left(\times 10^{9} / \mathrm{L}\right)$} \\
\hline Median (range) & $41.5(1.5-542)$ & $76(1.9-542)$ & 55 (1.6-198) & $15.3(1.5-99)$ & \\
\hline$<50 \times 10^{9} / \mathrm{L}$ & $41(50 \%)$ & $10(41.7 \%)$ & $19(45.2 \%)$ & $12(75 \%)$ & \multirow{2}{*}{0.065} \\
\hline$\geq 50 \times 10^{9} / \mathrm{L}$ & $41(50 \%)$ & $14(58.3 \%)$ & $23(54.8 \%)$ & $4(25 \%)$ & \\
\hline \multicolumn{6}{|l|}{ CNS infiltration at diagnosis } \\
\hline No & $72(87.8 \%)$ & $18(75 \%)$ & $40(95.2 \%)$ & $14(87.5 \%)$ & \multirow[t]{2}{*}{0.133} \\
\hline Yes & $10(12.2 \%)$ & $6(25 \%)$ & $2(4.8 \%)$ & $2(12.5 \%)$ & \\
\hline \multicolumn{6}{|l|}{ Immunophenotyping } \\
\hline B-ALL & $59(72 \%)$ & $16(66.7 \%)$ & $27(64.3 \%)$ & $16(100 \%)$ & \multirow[t]{2}{*}{0.02} \\
\hline T-ALL & $23(28 \%)$ & $8(33.3 \%)$ & $15(35.7 \%)$ & 0 & \\
\hline \multicolumn{6}{|l|}{ Site of relapse } \\
\hline Isolated medullary relapse & $37(45.1 \%)$ & $20(83.3 \%)$ & $13(31 \%)$ & $4(25 \%)$ & \multirow[t]{3}{*}{$<0.001$} \\
\hline Isolated extramedullary relapse & $34(41.5 \%)$ & $4(16.7 \%)$ & $22(52.4 \%)$ & $8(50 \%)$ & \\
\hline Combined & $11(13.4 \%)$ & 0 & $7(16.7 \%)$ & $4(25 \%)$ & \\
\hline
\end{tabular}

CNS: Central nervous system; TLC: Total leucocytic count

early and none of them relapsed late. The site of relapse differed significantly as well according to the timing of relapse. Other variables had no significant association as shown in Table 1.

\section{Response to reinduction}

The treatment outcome is shown in Figure 1. Forty-seven (57.3\%) patients achieved CR2, 21 (25.6\%) had reinduction failure and 14 (17.1\%) died during reinduction. Eight out of the 21patients with reinduction failure had $\geq 25 \%$ bone marrow response. Reinduction deaths were due to infectious complications (8 patients with fulminant pneumonia, 4 with acute gastroenteritis and typhlitis, and 2 with hypoalbuminemia and sepsis).

The relationship between reinduction outcome and patients' characteristics is shown in Table 2. Reinduction outcome differed significantly according to initial total leucocytic count (TLC), immunophenotyping, site of relapse and reinduction regimen. As regards the reinduction regimen, CR2 was far more common with regimen 2 than with regimen 1 ( $64.6 \%$ vs. $29.4 \%$, respectively; $p=0.006)$.

\section{Post-reinduction therapy}

Among the 7 patients who developed toxic deaths after the FLAG consolidation, 5 failed reinduction with IMR and 2 achieved CR2. The seven deaths were due to chest infection and sepsis.

During continuation treatment, 18 patients (22\%) developed $2^{\text {nd }}$ relapse within a median time of 11 months (range: 2- 26). There was no significant difference in the rate of $2^{\text {nd }}$ relapse between regimen 1 and $2(4 / 17$ [23.5\%] vs $14 / 65$ [21.5\%], $p=0.184$ ]. Second relapse was mainly CNS relapse in 12 patients. All patients who experienced a $2^{\text {nd }}$ relapse died due to disease progression within 4 months. While progression occurred mainly in the bone marrow in 13 patients who survived after reinduction failure. Myelosuppression was the most frequent cause of delay of intensive continuation therapy for $>4$ months in $33(54.1 \%)$ patients. Sixteen patients did not complete intensive continuation; 3 of them were referred for BMT and the others got relapse or progression. Treatment-related mortality in 8 (9.8\%) patients (all of them received regimen 2 ) after CR2 during continuation treatment and the causes were brain abscess (1), post-infectious Guillain Barre (1), $2^{\text {ry }}$ AML (2), fulminant bacterial pneumonia and sepsis (1) and organ dysfunction (3).

All nonfatal complications recovered except in 2 


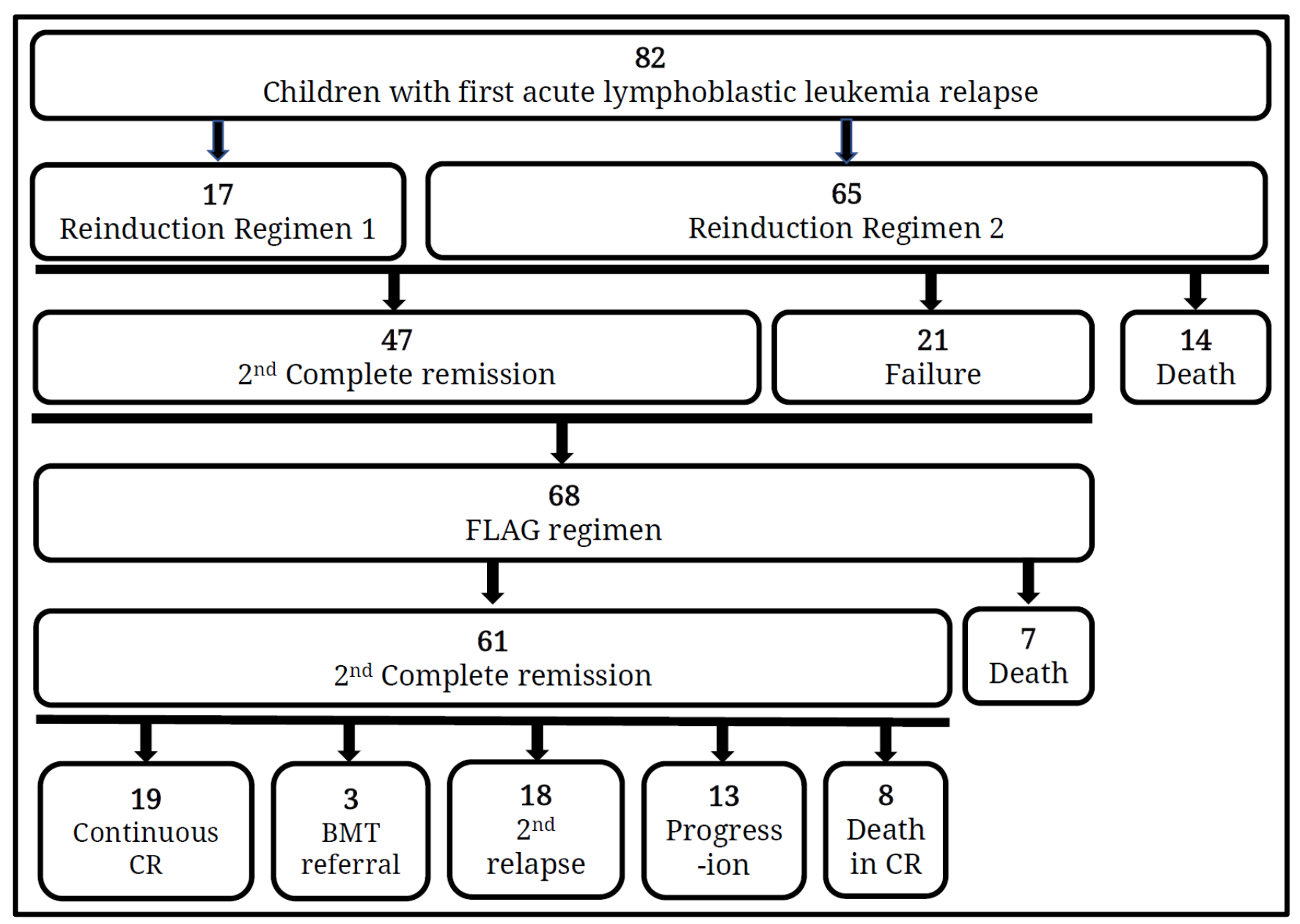

Figure 1: Flow diagram of management outcome of 82 children with first acute lymphoblastic leukemia relapse

Table 2: The relationship between reinduction outcome and variables

\begin{tabular}{|c|c|c|c|c|}
\hline \multirow[t]{2}{*}{ Variable } & \multicolumn{3}{|c|}{ Reinduction outcome } & \multirow[t]{2}{*}{$p$ value } \\
\hline & $\begin{array}{l}2^{\text {nd }} \text { complete remission } \\
(n=47)\end{array}$ & $\begin{array}{l}\text { Reinduction failure } \\
(n=21)\end{array}$ & $\begin{array}{l}\text { Reinduction death } \\
(n=14)\end{array}$ & \\
\hline \multicolumn{5}{|l|}{ Age at diagnosis (years) } \\
\hline$<10$ & $28(54.9 \%)$ & $13(25.5 \%)$ & $10(19.6 \%)$ & \multirow[t]{2}{*}{0.724} \\
\hline$\geq 10$ & $19(61.3 \%)$ & $8(25.8 \%)$ & $4(12.9 \%)$ & \\
\hline \multicolumn{5}{|l|}{ Initial TLC $\left(\times 10^{9} / \mathrm{L}\right)$} \\
\hline$<50$ & $31(75.6 \%)$ & $6(14.6 \%)$ & $4(9.8 \%)$ & \multirow[t]{2}{*}{0.004} \\
\hline$\geq 50$ & $16(39 \%)$ & $15(36.6 \%)$ & $10(24.4 \%)$ & \\
\hline \multicolumn{5}{|l|}{ Immunophenotyping } \\
\hline B-ALL & $34(57.6 \%)$ & $19(32.2 \%)$ & $6(10.2 \%)$ & \multirow[t]{2}{*}{0.009} \\
\hline T-ALL & $13(56.5 \%)$ & $2(8.7 \%)$ & $8(34.8 \%)$ & \\
\hline \multicolumn{5}{|l|}{ Timing of relapse } \\
\hline Very early & $10(41.7 \%)$ & $10(41.7 \%)$ & $4(16.7 \%)$ & \multirow[t]{3}{*}{0.747} \\
\hline Early & $25(59.5 \%)$ & $7(16.7 \%)$ & $10(23.8 \%)$ & \\
\hline Late & $12(75 \%)$ & $4(25 \%)$ & 0 & \\
\hline \multicolumn{5}{|l|}{ Site of relapse } \\
\hline Isolated medullary relapse & $16(43.2 \%)$ & $17(45.9 \%)$ & $4(10.8 \%)$ & \multirow[t]{3}{*}{0.003} \\
\hline Isolated extramedullary relapse & $22(64.7 \%)$ & $4(11.8 \%)$ & $8(23.5 \%)$ & \\
\hline Combined & $8(81.8 \%)$ & 0 & $2(18.2 \%)$ & \\
\hline \multicolumn{5}{|l|}{ Reinduction regimen } \\
\hline Regimen 1 & $5(29.4 \%)$ & $10(58.8 \%)$ & $2(11.8 \%)$ & \multirow[t]{2}{*}{0.006} \\
\hline Regimen 2 & $42(64.6 \%)$ & $11(16.9 \%)$ & $12(18.5 \%)$ & \\
\hline
\end{tabular}


patients; one had subacute encephalopathy and the other had epilepsy postcranial radiotherapy. Nineteen (23.2\%) patients were still living in continuous complete remission (CCR) for a median duration of 41 months (range: 21-77).

Disease-related death was more common in regimen 1 than regiment 2 (14 [82.4\%] patients vs. 25 [38.5\%], respectively), while treatment-related mortality was more common in regiment 2 than regiment 1 (22 [33.8\%] patients vs. 2 [11.76\%], respectively) ( $p=0.003)$.

\section{Survival outcome}

With a median follow-up of 39 months (range: 9141), the 2 -year OS rate was $30.2 \%$ (SE: 5), and the 2 -year EFS rate was 25.4\% (SE: 5) (Figure 2).

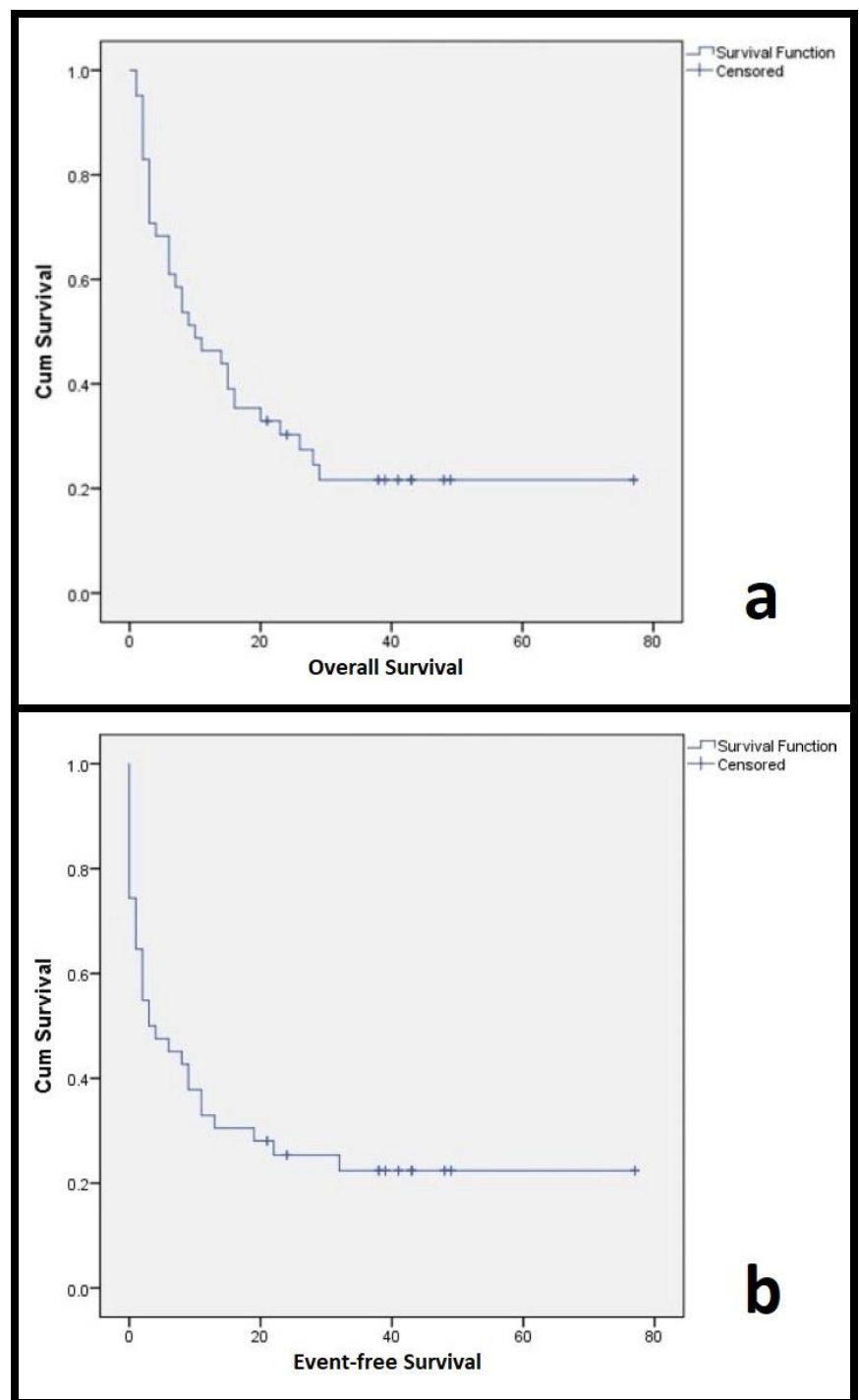

Figure 2: Kaplan- Meier curves of a) overall survival and $b$ ) event-free survival
The relationship between the studied variables and the 2-year overall and event-free survival rates is shown in Table 3.

The multivariate Cox regression analysis confirmed the unfavourable outcome of high initial TLC, reinduction regimen 1, IMR, and early / very early relapse on EFS with the highest hazard ratios were associated with IMR and very early relapse as shown in Table 4. Immunophenotyping had no impact on survival outcomes after fixing other prognostic factors (TLC, timing of relapse, site of relapse) except for that B-cell ALL had a significantly better OS than T-cell only if associated with favourable age $(p=0.026)$.

Reinduction regimen 2 improved significantly the EFS of very early relapse and early relapse patients than regimen 1 ( $p=0.011$ and 0.020). Event-free survival of late relapse did not differ significantly between regimens 1 and $2(p=0.170)$.

\section{Discussion}

We found that the overall outcome of children with relapsed ALL in our region is poor; however, good survival was observed in some of them. Timing of relapse was the most powerful predictor of outcome after relapse. Second CR and 2-year OS rates of patients with late relapse were $75 \%$ and 87.5\%, respectively. Adding anthracyclines to the reinduction treatment had a better impact on inducing CR2 and on OS and EFS of very early / early relapse but not late relapse. These findings can help us in designing the treatment strategy after relapse in children with ALL based on the timing of relapse.

Median time until relapse of our ALL patients was 27 months (range: $3-100$ ) with more than $80 \%$ of relapsed patients in the study were very early or early relapse. This is in agreement with others ${ }^{20-22}$.

Late relapse in our study did not involve older age ( $\geq 10$ years) or T-ALL, that reported by others 4 , ${ }^{23-25}$. It is well known that the majority of relapses occurs in the bone marrow ${ }^{4,21,26,27}$. While, in our study, the pattern of relapse has changed with an increase of IEMR to be near IMR ( $41.5 \%$ and $45.1 \%$ respectively) which was observed in an earlier study 12. This may be related to the lack of good identification and treatment of patients with CNS 2 criteria of de novo cases.

The overall 2-year OS and EFS rates $(30.2 \%$ and $25.4 \%$ ) in this study are much lower than those of developed countries where survival exceeds $50 \%{ }^{11}$, ${ }^{16}$. While we were very similar to the 3-year OS and 
Table 3: The relationship between variables and event-free and overall survival after first relapse in children with acute lymphoblastic leukemia

\begin{tabular}{|c|c|c|c|c|}
\hline Variable & 2-year EFS rate (SE) & $p$ value & 2-year OS rate (SE) & $p$ value \\
\hline \multicolumn{5}{|l|}{ Age at initial diagnosis (years) } \\
\hline$<10$ & $30.9(0.06)$ & \multirow[t]{2}{*}{0.340} & $38.8(0.07)$ & \multirow[t]{2}{*}{0.099} \\
\hline$\geq 10$ & $16.1(0.07)$ & & $16.1(0.07)$ & \\
\hline \multicolumn{5}{|l|}{ Sex } \\
\hline Male & $26.6(0.07)$ & \multirow[t]{2}{*}{0.131} & $31.7(0.08)$ & \multirow[t]{2}{*}{0.257} \\
\hline Female & $23.8(0.07)$ & & $28.6(0.07)$ & \\
\hline \multicolumn{5}{|l|}{ Initial TLC $\left(\times 10^{9} / \mathrm{L}\right)$} \\
\hline$<50$ & $41.5(0.08)$ & \multirow[t]{2}{*}{$<0.001$} & $46.3(0.08)$ & \multirow[t]{2}{*}{0.002} \\
\hline$\geq 50$ & $7.3(0.05)$ & & $13(0.06)$ & \\
\hline \multicolumn{5}{|l|}{ Immunophenotyping } \\
\hline B-ALL & $27.1(0.06)$ & 0.969 & $33.9(0.06)$ & \multirow[t]{2}{*}{0.281} \\
\hline T-ALL & $18.3(0.09)$ & & $18.3(0.09)$ & \\
\hline \multicolumn{5}{|l|}{ CNS infiltration at initial diagnosis } \\
\hline No & $26.1(0.05)$ & \multirow[t]{2}{*}{0.449} & $31.7(0.06)$ & \multirow[t]{2}{*}{0.772} \\
\hline Yes & $20(0.1)$ & & $20(0.1)$ & \\
\hline \multicolumn{5}{|l|}{ Timing of relapse } \\
\hline Very early & 0 & \multirow[t]{3}{*}{$<0.001$} & 0 & \multirow[t]{3}{*}{$<0.001$} \\
\hline Early & $25.3(0.07)$ & & $25.3(0.07)$ & \\
\hline Late & $62.5 \pm 0.1$ & & $87.5(0.08)$ & \\
\hline \multicolumn{5}{|l|}{ Site of relapse } \\
\hline Isolated medullary relapse & $10.8(0.05)$ & \multirow[t]{3}{*}{$<0.001$} & $10.8(0.05)$ & \multirow[t]{3}{*}{$<0.001$} \\
\hline Isolated extramedullary relapse & $22.1(0.07)$ & & $34.3(0.08)$ & \\
\hline Combined & $81.8(0.1)$ & & $81.3(0.1)$ & \\
\hline \multicolumn{5}{|l|}{ Reinduction regimen } \\
\hline Regimen 1 & $5.9(0.06)$ & \multirow[t]{2}{*}{0.011} & $17.6(0.09)$ & \multirow[t]{2}{*}{0.311} \\
\hline Regimen 2 & $30.5(0.06)$ & & $33.6(0.06)$ & \\
\hline
\end{tabular}

EFS: Event free survival, OS: Overall survival, SE: Standard error, TLC: Total leucocytic count, CNS: Central nervous system

Table 4: Multivariate Cox regression analysis of event free survival of 82 children with first acute lymphoblastic leukemia relapse

\begin{tabular}{|c|c|c|c|c|}
\hline Variable & $n$ & Hazard ratio & 95\% Confidence interval & $p$ value \\
\hline \multicolumn{5}{|l|}{ Total leucocytic count at initial diagnosis } \\
\hline$\geq 50$ & 41 & ref & & \\
\hline$<50$ & 41 & 0.555 & $0.325-0.947$ & 0.031 \\
\hline \multicolumn{5}{|l|}{ Reinduction regimen } \\
\hline Regimen 2 & 65 & ref & & \\
\hline Regimen 1 & 17 & 1.905 & $1.041-3.487$ & 0.037 \\
\hline \multicolumn{5}{|l|}{ Relapse site } \\
\hline Combined relapse & 11 & ref & & \\
\hline Isolated medullary relapse & 37 & 6.479 & $1.401-29.958$ & 0.017 \\
\hline Isolated extramedullary relapse & 34 & 4.059 & $0.920-17.911$ & 0.064 \\
\hline \multicolumn{5}{|l|}{ Timing of relapse } \\
\hline Late & 16 & ref & & \\
\hline Very early & 24 & 5.599 & $2.053-15.271$ & 0.001 \\
\hline Early & 42 & 2.971 & $1.122-7.866$ & 0.028 \\
\hline
\end{tabular}

EFS rates of $31.5 \%$ and $26.2 \%$ of relapsed ALL patients in Central America who were treated with curative intent ${ }^{6}$. It should be noted that many factors exist making the comparison difficult, including many treatment protocols used for relapsed patients with varying type and intensity of chemotherapy. Also, most of the protocols in developed countries incorporated the use of BMT and targeted therapy in addition to the availability of adequate supportive care. 
Although the reported survival rates in our study are low, the 2-year OS and EFS for late relapse of $87.5 \%$ and $60 \%$ are comparable to the 3-year EFS and OS of $63.6 \%$ and $72.3 \%$ for late B- cell precursor bone marrow relapse in the AALL0433 trial of COG ${ }^{28}$, 3year progression-free survival of $60 \%$ and OS of $72 \%$ in the UK ALLR3 trial ${ }^{29}$ and 64\% 5-year EFS in Relapse ALL 98 Protocol Based on the Dutch COG ${ }^{3}$. While the poor 2-year survival for those with very early and early relapse in our study (0 and 25\%) were comparable to EFS of other results (5\% to $18 \%$ ) $7,8,30$.

The universal favourable outcome of late relapse irrespective of the treatment strategies, confirms that late relapse retains the chemo-sensitivity of the original clone as they arise from a common precursor ${ }^{11}$.

The poor 2-year OS of IMR of our cohort (10.8\%) is matched with most studies as the survival of IMR is worse than IEMR with an intermediate outcome of combined relapse $4,5,16$. Combined relapse was unexpectedly associated with the most favourable survival (81.3\%) that exceeded the survival of IEMR (34.3\%). This may be related to the small number of patients with combined relapse and most of them showed favourable criteria (B-ALL in 8/11, favourable TLC in 9/11, no very early relapse, the least TRM) in addition to the early local control of the extramedullary sites that are hypothesized to be the origin of combined bone marrow relapse ${ }^{14}$.

While the BFM and United Kingdom ALL R3 study groups' risk stratifications are based on either the relapse B-cell or T-cell ALL ${ }^{11}{ }^{13}$, the unfavourable prognosis of T-immunophenotype in patients with ALL relapse varied between studies if it is independent or differs according to time or site of relapse. The BFM group and SJCRH investigators reported that medullary T-cell ALL relapse has a much worse prognosis than B-cell ALL 13, 22. Rheingold et al. reported that the survival after relapse differed according to the site and certain time points of relapse ${ }^{4}$. Others demonstrated that the unfavourable outcome of T- immunophenotype is not related to the site or time of relapse $5,7,26$. Oskarsson et al. reported its poor outcome only if associated with hyperleukocytosis at diagnosis ${ }^{16}$. Our results did not show a significant difference between B-cell and T-cell ALL either overall or by time or site of relapse. This may be due to the very small number of each group if classified by time and site.

The rate of CR2 achievement of $57.3 \%$ (41.7\%, $59.5 \%, 75 \%$ for very early, early and late relapse respectively) is much lower than that reported in the literature ${ }^{8,30}$. This may be due to the low CR2 among patients who received regimen 1 (29.4\%) in addition to the high rate of deaths due to toxicity during reinduction.

In our study, the rate of reinduction deaths of $17.1 \%$ is much higher than the $4 \%$ in COG AALL1331 reinduction regimen using mitoxantrone $31,32$. Patients during reinduction should be hospitalized until count recovery with antibacterial, antifungal, and Pneumocystis jirovecii prophylaxis due to the high rate of infection as recommended by Hanger and Raetz's strategy ${ }^{33}$.

The overall TRM in our study $(29.3 \%)$ is much higher than that reported by others. It was $5 \%$ (4 of 76) in the 9412 protocol ${ }^{34}, 17.4 \%$ and $9.7 \%$ in the idarubicin and mitoxantrone arms of the ALL R3 protocol ${ }^{11}, 4 \%$ (5 of 124) in the AALL01P2 study protocol of $\mathrm{COG}^{8}$ and $15 \%$ (37 of 246) in patients treated with ALL-REZ BFM $90{ }^{5}$. When compared to other low middle-income countries, $29.7 \%$ of patients suffered treatment-related toxicities, which is similar to our results ${ }^{6}$.

The treatment of the studied patients included the use of dexamethasone instead of prednisone which affects controlling CNS disease through penetrating the blood brain barrier and intensive use of asparaginase which also has a role in CNS control. Moreover, the treatment included the use of new chemotherapeutic agents that were not used for newly diagnosed cases to overcome the resistance and showed a marked response. Mitoxantrone was reported to have an excellent effect on the outcome of ALL relapse patients through its delayed cytotoxic effect ${ }^{11}$. This can explain why all TRM during continuation was associated with regimen 2 despite the insignificant difference of reinduction deaths between the two regimens. A further study of the separate effect of mitoxantrone and daunorubicin is needed. Fludarabine, in the FLAG combination, produces a synergistic effect when combined with cytarabine and granulocyte colony-stimulating factor (G-CSF). The successful use of this regimen has been established in children for the treatment of refractory and relapsed ALL with minimal toxicity ${ }^{9}$, 35. FLAG induced CR in all patients with reinduction failure in our cohort.

\section{Conclusion}

The survival of children with ALL after the first relapse at our centres has improved through the treatment with a uniform protocol but the outcome is still poorer than in developed countries. The 
timing and site of relapse are strong predictors of the outcome and the addition of anthracyclines for those with early and very relapses has better control on leukemia, but the treatment-related toxicities are high. Therefore, we suggest designing a stratified treatment with the coverage of meticulous supportive care. Being a retrospective study is a limitation with the unequal representation of the two reinduction regimens studied. Conventional chemotherapeutic drugs still have a role in the treatment of ALL relapse with the incorporation of new drugs not used at initial treatment is recommended until treatment with targeted therapies becomes a reality.

\section{Acknowledgment}

None.

\section{Authors' contribution}

Conception or design: All authors; Acquisition, analysis or interpretation of data: All authors; Drafting or revising the manuscript: All authors; Approval of the manuscript version to be published: All authors; Agreement to be accountable for all aspects of the work: All authors.

\section{Conflict of interest}

The authors declare that they have no conflict of interest to disclose.

\section{Data a vailability}

Deidentified individual participant data used to produce the results of this study are available from the corresponding author (AS) upon request.

\section{Ethical considerations}

This study was approved by the Institutional Review Board of South Egypt Cancer Institute, Assiut University, Assiut, Egypt (approval No.: 152).

\section{Funding \\ None.}

\section{Study registration}

None.

\section{References}

1. Pui CH, Yang JJ, Hunger SP, et al. Childhood acute lymphoblastic leukemia: progress through collaboration. J Clin Oncol. 2015; 33(27): 2938-2948.

2. Hunger SP, Lu X, Devidas M, et al. Improved survival for children and adolescents with acute lymphoblastic leukemia between 1990 and 2005: a report from the children's oncology group. J Clin Oncol. 2012; 30(14): 1663-1669.

3. van den Berg H, de Groot-Kruseman HA, DamenKorbijn CM, de Bont ES, Schouten-van Meeteren AY, Hoogerbrugge PM. Outcome after first relapse in children with acute lymphoblastic leukemia: a report based on the Dutch Childhood Oncology Group
(DCOG) relapse all 98 protocol. Pediatr Blood Cancer. 2011; 57(2) :210-216.

4. Rheingold SR, Ji L, Xu X, et al. Prognostic factors for survival after relapsed acute lymphoblastic leukemia (ALL): A Children's Oncology Group (COG) study. J Clin Oncol. 2019; 37(Suppl 15): 10008.

5. Tallen G, Ratei R, Mann G, et al. Long-term outcome in children with relapsed acute lymphoblastic leukemia after time-point and site-of-relapse stratification and intensified short-course multidrug chemotherapy: results of trial ALL-REZ BFM 90. J Clin Oncol. 2010; 28(14): 2339-2347.

6. Marjerrison S, Antillon F, Fu L, et al. Outcome of children treated for relapsed acute lymphoblastic leukemia in Central America. Cancer. 2013; 119(6): 1277-1283.

7. Nguyen K, Devidas M, Cheng S-C, et al. Factors influencing survival after relapse from acute lymphoblastic leukemia: a Children's Oncology Group study. Leukemia. 2008; 22(12): 2142-2150.

8. Raetz EA, Borowitz MJ, Devidas M, et al. Reinduction platform for children with first marrow relapse of acute lymphoblastic leukemia: A Children's Oncology Group Study. J Clin Oncol. 2008; 26(24): 3971-3978.

9. Hijiya N, Stewart CF, Zhou Y, et al. Phase II study of topotecan in combination with dexamethasone, asparaginase, and vincristine in pediatric patients with acute lymphoblastic leukemia in first relapse. Cancer. 2008; 112(9): 1983-1991.

10. Rivera GK, Hudson MM, Liu Q, et al. Effectiveness of intensified rotational combination chemotherapy for late hematologic relapse of childhood acute lymphoblastic leukemia. Blood. 1996; 88(3): 831-837.

11. Parker C, Waters R, Leighton $C$, et al. Effect of mitoxantrone on outcome of children with first relapse of acute lymphoblastic leukaemia (ALL R3): an open-label randomised trial. Lancet. 2010; 376(9757): 2009-2017.

12. Shibl A, Sayed H, Ali A, Mohamed D, Abdelhamid O. Long term survival outcome of childhood acute lymphoblastic leukemia treated with modified TXIIIB protocol at South Egypt Cancer Institute. Int J Cancer Biomed Res. 2021; 5(3): 121-132.

13. Borgmann A, von Stackelberg A, Hartmann R, et al. Unrelated donor stem cell transplantation compared with chemotherapy for children with acute lymphoblastic leukemia in a second remission: a matched-pair analysis. Blood. 2003; 101(10): 38353839.

14. Fuster JL. Current approach to relapsed acute lymphoblastic leukemia in children. World J Hematol. 2014; 3(3): 49-70.

15. Oskarsson T, Söderhäll S, Arvidson J, et al. Treatmentrelated mortality in relapsed childhood acute lymphoblastic leukemia. Pediatr Blood Cancer. 2018; 65(4): e26909.

16. Oskarsson T, Söderhäll S, Arvidson J, et al. Relapsed childhood acute lymphoblastic leukemia in the 
Nordic countries: prognostic factors, treatment and outcome. Haematologica. 2016; 101(1): 68-76.

17. Pui CH, Sandlund JT, Pei D, et al. Improved outcome for children with acute lymphoblastic leukemia: results of Total Therapy Study XIIIB at St Jude Children's Research Hospital. Blood. 2004; 104(9): 2690-2696.

18. Schrappe M, Reiter A, Zimmermann M, et al. Longterm results of four consecutive trials in childhood ALL performed by the ALL-BFM study group from 1981 to 1995. Leukemia. 2000; 14(12): 2205-2222.

19. Pui CH, Campana D, Pei D, et al. Treating childhood acute lymphoblastic leukemia without cranial irradiation. N Engl J Med. 2009; 360(26): 2730-2741.

20. Saarinen-Pihkala UM, Heilmann C, Winiarski J, et al. Pathways through relapses and deaths of children with acute lymphoblastic leukemia: role of allogeneic stem-cell transplantation in Nordic data. J Clin Oncol. 2006; 24(36): 5750-5762.

21. Roy A, Cargill A, Love S, et al. Outcome after first relapse in childhood acute lymphoblastic leukaemialessons from the United Kingdom R2 trial. Br J Haematol. 2005; 130(1): 67-75.

22. Rivera GK, Zhou Y, Hancock ML, et al. Bone marrow recurrence after initial intensive treatment for childhood acute lymphoblastic leukemia. Cancer. 2005; 103(2): 368-376.

23. Teachey DT, Hunger SP. Predicting relapse risk in childhood acute lymphoblastic leukaemia. $\mathrm{Br} \mathrm{J}$ Haematol. 2013; 162(5): 606-620.

24. Szczepanski T, van der Velden VH, Waanders E, et al. Late recurrence of childhood T-cell acute lymphoblastic leukemia frequently represents a second leukemia rather than a relapse: first evidence for genetic predisposition. J Clin Oncol. 2011; 29(12): 1643-1649.

25. Hastings C, Gaynon PS, Nachman JB, et al. Increased post-induction intensification improves outcome in children and adolescents with a markedly elevated white blood cell count ( $\geq 200 \times 109 / 1$ ) with T cell acute lymphoblastic leukaemia but not B cell disease: a report from the $\mathrm{C}$ hildren's $\mathrm{O}$ ncology $\mathrm{G}$ roup. $\mathrm{Br} \mathrm{J}$ Haematol. 2015; 168(4): 533-546.

26. Reismüller B, Attarbaschi A, Peters C, et al. Long-term outcome of initially homogenously treated and relapsed childhood acute lymphoblastic leukaemia in Austria-A population-based report of the Austrian Berlin-Frankfurt-Münster (BFM) Study Group. Br J Haematol. 2009; 144(4): 559-570.
27. Chessells JM, Veys P, Kempski H, et al. Long-term follow-up of relapsed childhood acute lymphoblastic leukaemia. Br J Haematol. 2003; 123(3): 396-405.

28. Lew G, Chen Y, Lu X, et al. Outcomes after late bone marrow and very early central nervous system relapse of childhood B-acute lymphoblastic leukemia: a report from the Children's Oncology Group phase III study AALL0433. Haematologica. 2021; 106(1): 4655.

29. Parker C, Krishnan S, Hamadeh L, et al. Outcomes of patients with childhood B-cell precursor acute lymphoblastic leukaemia with late bone marrow relapses: long-term follow-up of the ALLR3 openlabel randomised trial. Lancet Haematol. 2019; 6(4): e204-e16.

30. Ko RH, Ji L, Barnette P, et al. Outcome of patients treated for relapsed or refractory acute lymphoblastic leukemia: a Therapeutic Advances in Childhood Leukemia Consortium study. J Clin Oncol. 2010; 28(4): 648-654.

31. Hogan LB, Bhatla T, Rheingold S, et al. Induction toxicities are more frequent in young adults compared to children treated on the Children's Oncology Group (COG) first relapse b-lymphoblastic leukemia clinical trial AALL1331. Blood. 2018; 132(Suppl 1): 1382.

32. Brown PA, Ji L, Xu X, et al. A randomized phase 3 trial of blinatumomab vs. chemotherapy as postreinduction therapy in high and intermediate risk (HR/IR) first relapse of b-acute lymphoblastic leukemia (B-ALL) in children and adolescents/young adults (AYAs) demonstrates superior efficacy and tolerability of blinatumomab: A report from Children's Oncology Group study AALL1331. Blood. 2019; 134(Suppl 2): LBA-1.

33. Hunger SP, Raetz EA. How I treat relapsed acute lymphoblastic leukemia in the pediatric population. Blood. 2020; 136(16): 1803-1812.

34. Barredo JC, Devidas M, Lauer SJ, et al. Isolated CNS relapse of acute lymphoblastic leukemia treated with intensive systemic chemotherapy and delayed CNS radiation: a pediatric oncology group study. J Clin Oncol. 2006; 24(19): 3142-3149.

35. McCarthy A, Pitcher L, Hann I, Oakhill A. FLAG (fludarabine, high-dose cytarabine, and G-CSF) for refractory and high-risk relapsed acute leukemia in children. Med Pediatr Oncol. 1999; 32(6): 411-415. 